\title{
SDMtoolbox 2.0: the next generation python-based GIS toolkit for landscape genetic, biogeographic and species distribution model analyses
}

\author{
Jason L Brown ${ }^{\text {Corresp., }}{ }^{1}$, Joseph R Bennett ${ }^{1}$, Connor M French ${ }^{1}$ \\ ${ }^{1}$ Department of Zoology, Cooperative Wildlife Research Laboratory, Southern Illinois University at Carbondale, Carbondale, IL, USA \\ Corresponding Author: Jason L Brown \\ Email address: jason.brown@siu.edu
}

SDMtoolbox 2.0 is a software package for spatial studies of ecology, evolution, and genetics. The release of SDMtoolbox 2.0 allows researchers to use the most current ArcGIS software and MaxEnt software, and reduces the amount of time that would be spent developing common solutions. The central aim of this software is to automate complicated and repetitive spatial analyses in an intuitive graphical user interface. One core tenant facilitates careful parameterization of species distribution models (SDMs) to maximize each model's discriminatory ability and minimize overfitting. This includes carefully processing of occurrence data, environmental data and model parameterization. This program directly interfaces with MaxEnt, one of the most powerful and widely used species distribution modeling software programs, although SDMtoolbox 2.0 is not limited to species distribution modeling or restricted to modeling in MaxEnt. Many of the SDM preand post-processing tools have 'universal' analogs for use with any modeling software. The current version contains a total of 79 scripts that harness the power of ArcGIS for macroecology, landscape genetics, and evolutionary studies. For example, these tools allow for biodiversity quantification (such as species richness or corrected weighted endemism), generation of least-cost paths and corridors among shared haplotypes, assessment of the significance of spatial randomizations, and enforcement of dispersal limitations of SDMs projected into future climates--- to only name a few functions contained in SDMtoolbox 2.0. Lastly, dozens of generalized tools exists for batch processing and conversion of GIS data types or formats, which are broadly useful to any ArcMap user. 
1 TITLE: SDMtoolbox 2.0: the next generation python-based GIS toolkit for landscape genetic,

2 biogeographic and species distribution model analyses

3 AUTHORS: Jason L. Brown*1, Joseph R. Bennett ${ }^{1}$, Connor M. French ${ }^{1}$

4 Affiliations: Southern Illinois University, Department of Zoology, Cooperative Wildlife Research

5 Laboratory, Carbondale, IL, 62901, USA

6 Corresponding Author: Jason L. Brown, $\underline{\text { *sdmtoolbox.help@gmail.com }}$

7

8 Note to editor and reviewers:

9 As follows is the main body of the manuscript and an extensive user guide for this software. Also included are links to: example data (same data used in the user guide), the software, and an install guide

11 (how to quickly install this). Note that reviewers must have access to ArcGIS 10.x with a Standard or

12 Advance license level with Spatial Analyst Extension. 


\section{ABSTRACt}

SDMtoolbox 2.0 is a software package for spatial studies of ecology, evolution, and genetics. The release of SDMtoolbox 2.0 allows researchers to use the most current ArcGIS software and MaxEnt software, and reduces the amount of time that would be spent developing common solutions. The central aim of this software is to automate complicated and repetitive spatial analyses in an intuitive graphical user interface. One core tenant facilitates careful parameterization of species distribution models (SDMs) to maximize each model's discriminatory ability and minimize overfitting. This includes carefully processing of occurrence data, environmental data and model parameterization. This program directly interfaces with MaxEnt, one of the most powerful and widely used species distribution modeling software programs, although SDMtoolbox 2.0 is not limited to species distribution modeling or restricted to modeling in MaxEnt. Many of the SDM pre- and post-processing tools have 'universal' analogs for use with any modeling software. The current version contains a total of 79 scripts that harness the power of ArcGIS for macroecology, landscape genetics, and evolutionary studies. For example, these tools allow for biodiversity quantification (such as species richness or corrected weighted endemism), generation of least-cost paths and corridors among shared haplotypes, assessment of the significance of spatial randomizations, and enforcement of dispersal limitations of SDMs projected into future climates--- to only name a few functions contained in SDMtoolbox 2.0. Lastly, dozens of generalized tools exists for batch processing and conversion of GIS data types or formats, which are broadly useful to any ArcMap user. 
53

54

55

56

57

\section{INTRODUCTION}

SDMtoolbox is a python-based ArcGIS toolbox for spatial studies of ecology, evolution and genetics. SDMtoolbox consists of a series of python scripts (79 and growing) designed to automate complicated spatial analysis in ArcMap (ESRI) and python. Since SDMtoolbox's first release (Brown 2014), in April 2014 , the program has been download over 20,000 times by users in 160 countries (from every subcontinent) and cited over 147 times. Surveying SDMtoolbox's citations, most users (87\%) used this toolkit for the preparation of SDM files, running SDMs and processing of SDM results. Since the first publication (Brown 2014), SDMtoolbox has been under continuous development and expansion. SDMtoolbox remains a free comprehensive python-based toolbox for macroecology, landscape genetic and evolutionary studies to be used with ArcGIS 10.0 (or higher) with a Standard or Advance License and the Spatial Analyst extension. The toolkit simplifies many GIS analyses required for species distribution modelling and other spatial ecological analyses, alleviating the need for repetitive and time-consuming climate data pre-processing and post-SDM analyses.

\section{Methods}

SDMtoolbox is written in Python (v2.7) and incorporates python libraries from ArcPy (ArcGIS 10.0-10.5), NumPy and SciPy that are incorporated into a single toolbox for use by ArcGIS 10.0-10.5 users. One tool, the Run MaxEnt: Spatially Jackknife tool, outputs java code batch file format to run the MaxEnt program.

\section{RESULTS}

After the release of SDMtoolbox v1, we updated 28 (of 59) of the original tools to improve user performance and maximize compatibility with newer versions of ArcGIS 10.3-10.5 and the recent open source version of MaxEnt (version 3.4 or higher; Phillips et al. 2017). For several tools this required completely recoding the analysis pipeline (Table 1 ). In addition to updating many tools, we provide 20 new tools, increasing the total tools to 79 (Table 2). The adaption of the code in the Run MaxEnt: Spatially Jackknife tool for MaxEnt 3.4 enables output models in cloglog format and enabling/disabling use of threshold feature class in spatial jackknifing process. Following are brief overviews of the new tools, organized by major tool category (Brown 2014)

\section{Biodiversity Measurements}


81 This suite of tools calculates spatial biodiversity patterns. One of two new tools facilitate a quantitative 82 method for locating hotspots of endemism, and is called Categorical Analysis of Neo- and Paleo83 Endemism (CANAPE; Mishler et al. 2014). These analyses are able to classify neo-endemic and paleoendemic species, young taxon and an old taxon with a restricted distribution, respectively. This method assesses the significance of branch lengths among taxa that are either significantly shorter (neo) or significantly longer (paleo) than other areas in the landscape. The randomizations are performed in the standalone program Biodiverse (Laffan et al. 2010) and outputs are input into SDMtoolbox 2.0, which performs the series of analyses for categorization of significant neo- and paleo-endemic areas. A second, similar tool categorizes significance of randomizations done in Biodiverse and is applicable to any randomization performed in Biodiverse (Laffan et al. 2010).

\section{Landscape Connectivity}

This suite of tools measure landscape connectivity among populations. One prevalent method to do this is to estimate least-cost paths (LCPs) among sites (e.g., Ray 2005, McRae and Beier 2007). The new tool added to this category measures LCPs and output distance matrices of the LCP distance and the alongpath-cost of each LCP.

\section{Species Distribution Modeling tools}

Many of the python scripts contained in SDMtoolbox were initially written for species distribution modelling. Two new tools have been added to this group. The first tool splits a binary SDM by userinput clade relationships. This is done by dividing the landscape by Voronoi polygons generated from input localities and then each polygon is grouped by the clade relationship. The polygon of each clade relationship is used to mask the input SDM and output is the proportion of the SDM corresponding to that clade distribution. This tool is useful for dividing species distributions by their phylogenetic relationships.

The second species distribution modeling tool provides a novel method for creating bias files for use in MaxEnt (see discussion for overview and importance of Bias files in MaxEnt). Often distributions can be largely separated by unsuitable habitat. The second tool limits the selection of background points to an area encompassed by a buffered regional convex-hull based on species occurrences. The area of background selection is intermediate between the buffered minimum-convex polygon tool (which can include considerable area between distance localities) and the distance from observed localities tool (which can be quite restrictive). A main parameter for this tool is the alpha parameter that depicts the 
111 distance where points are aggregated into a convex-hull. Using this tool bias file be represent several

112 disjunct buffered polygons. Larger values will result in areas of background selection more similar to a

113 buffered minimum-convex polygon (MCP) and smaller values more similar to outputs from the distance

114 from observed localities tool. Generally speaking, this tool typically results in regional buffered MCP

115 based on spatial clusters of points.

\section{Basic Tools}

117 Most of the basic tools facilitate batch processing or conversion of data required for spatial analyses.

118 Fourteen new tools have been added to this group. Given a long standing ArcMap issue associated with extracting raster value to points in ArcGIS, we created a tool that achieves the same end product using a different data pipeline that works in most cases where the ArcMap's native tool failed. Several of the new tools facilitate conversion of rasters to other raster formats (i.e. NetCDF or float values). Other new

122 tools batch project or define the projection of input rasters and shapefiles to any projection. Another set 123 of tools facilitate batch exportation of images or aids in the display of red-green-blue (RGB) bands in

124 multiband rasters (e.g., which band is applied to red spectrum) by exporting images of all color permutations of RGB band combinations.

126 The default method for upscaling rasters (making a raster a coarser spatial resolution) is to resample by 127 selecting the value of a single pixel within an area of reduction or by interpolating values between the 128 nearest pixels associated with the centroid of each new pixel. In biological data, this can overlook considerable variation within the higher resolution data that is not incorporated in the coarser up-scaled data. Careful upscaling of data is extremely important when generating environmental files for SDMs. One new tools aims to provide more flexibility and precision when upscaling rasters by using input spatial statistics, such as mean, majority, median, and minority to name of few.

\section{Discussion}

As previously mentioned, a large proportion of SDMtoolbox v1 users cite using the program for assisting species distribution modeling. One of the reasons for this broad use appears to be the software's balance of user control with the simplicity of the graphical user interface (GUI) interface and automated model parameterization. SDMtoolbox facilities modeling from start to finish, by aiding compilation of occurrence and environmental data, and spatially vetting occurrence data to reduce spatial biases in occurrence records. Lastly, it rigorously parametrizes each MaxEnt model by performing spatial jacking 
141 itemized points below). This parametrization approach can allow the selection of model settings that

142 have high discriminatory ability and a model that minimizes overfitting to noise as well as to the spatial

143 biases in occurrence data and corresponding environmental biases (Radosavljevic \& Anderson 2014).

144 Low overfitting and high discriminatory ability are the two main desired qualities of a 'good' species

145 distribution model (Lobo et al. 2008, Peterson et al. 2011, Warren and Seifert 2011). Discriminatory

146 ability characterizes the ability of the model to distinguish suitable from unsuitable areas and is typically

147 measured with the area under the curve of the receiver operating characteristic plot (AUC/ROC)

148 (Peterson et al. 2011) output from MaxEnt (and other programs). Overfitting is the tendency of a model

149 to fit the random error (or any bias in the sample) rather than the true relationship between the

150 calibration records and predictor variables. Often, over-fit models predict the calibration data very well,

151 but perform poorly on other data sets. Overfitting is typically assessed with the false negative rate, also

152 called omission error rate (OER henceforth). With an appropriately selected threshold converting a

153 continuous prediction into a binary one, OERs indicate the proportion of presences incorrectly classified

154 as falling into unsuitable areas (typically resulting from a prediction that is too tightly fit to the

155 conditions at calibration localities; Anderson et al. 2003). The best model output from SDMtoolbox is

156 the model with the lowest OER, and of those models, if multiple, the model with the highest AUC.

157 Lastly, if a model has an identically low OER and high AUC values, the feature class complexity is

158 accounted for selecting the model with the lowest complexity. If there is a single model with the lowest

159 OR, AUC values should be assessed in the MaxEnt outputs post-hoc and used as an independent metric

160 of performance resulting from model tuning.

161 The primary ways SDMtoolbox minimizes model overfitting and properly parameterizes each species

162 distribution model it creates (vs. using only MaxEnt without SDMtoolbox) are discussed in the following

163 paragraphs. The first example concerns with curation of occurrence records. To perform well, most

164 SDM methods require input-occurrence data to be spatially independent. However, researchers often

165 introduce environmental biases into their SDMs from spatially autocorrelated occurrence points. It is

166 important to eliminate spatial clusters of localities for model calibration and evaluation. When spatial

167 clusters of localities exist, often models are over-fit towards environmental biases (reducing the model's

168 ability to predict spatially independent data) and model performance values are inflated (Fig. 1D; Veloz

169 2009; Hijimans et al. 2012; Boria et al. 2014). In SDMtoolbox, this can be done in several ways, using the

170 Spatially Rarefy Occurrence Data for SDMs (reduce spatial autocorrelation) tool set. 
171 The second way SDMtoolbox minimizes model overfitting of each species distribution model it creates is

172 by carefully controlling the background selection by using bias files. Bias files control where background 173 points are selected and thereby avoid habitats greatly outside of a species' known occurrence.

174 Background points are meant to be compared with presence data to help identify the environmental 175 conditions under which a species can potentially occur. Typically, background points are selected within 176 a large rectilinear area. Within such areas, environmentally suitable but uncolonized or 177 biogeographically isolated habitat often exists. The selection of background points within these habitats 178 increases commission errors (false positives). As a result, the 'best' performing model tends to be over179 fitted because the selection criterion favors a model that fails to predict the species in the un-colonized 180 climatically suitable habitat (Anderson \& Raza 2010, Barbet-Massin et al. 2012). The likelihood that 181 suitable unoccupied habitats are included in background sampling increases with distance from the 182 realized range of the species. Thus, a larger study of spatial extent can lead to the selection of a higher 183 proportion of less informative background points (Barbet-Massin et al. 2012). Such issues are 184 ameliorated by being more selective in the choice of background points in MaxEnt (Barve et al. 2011; 185 Merow et al. 2013). In SDMtoolbox, this can be done several ways, using the Gaussian Kernel Density of 186 Sampling Localities, Sample by Buffered Local Adaptive Convex-Hull, Sample by Buffered MCP, and Sample by Distance from Obs. Pts. tools (Fig 1G).

The MaxEnt program attempts to limit model complexity during parameterization by controlling model regularization. This regularization imposes a penalty for each term included in the model and for higher weights given to a term (Phillips et al., 2006; Anderson \& Gonzalez, 2011). The current release of MaxEnt implements a regularization multiplier, which is a user-specified coefficient that is applied to the value of the respective beta parameters of each feature class incorporated into the model (Philips et al. 2006). This regularization multiplier alters the overall level of regularization rather than changing each beta parameter individually. The default setting in MaxEnt is a value of 1. Researchers have reported that regularization multipliers as high as 2.0 to 4.0 were necessary to reduce overfitting resulting from lower regularization multiplier values (Radosavljevic \& Anderson 2014). Qualitative assessments of the geographical predictions reiterate this conclusion (Radosavljevic \& Anderson 2014). To evaluate and compare multiple regularization parameters, in absence of SDMtoolbox, MaxEnt requires users are to run multiple instances of the program and then manually compare model performance statistics (i.e. OR and AUC). SDMtoolbox v2.0 allows you to input a range of regularization multipliers and automatically selects the value resulting in the best model (via the process clarified in the first paragraph of the Discussion). 
203 Another key parameter in a MaxEnt model is the feature class, which determines the kinds of

204 constraints allowed in a model. A feature is a function of user input environmental variables and can be

205 any single one or various combination of six feature classes implemented: linear (L), quadratic (Q),

206 product $(P)$, threshold $(T)$, hinge $(H)$ or category indicator $(C)$ ( Phillips et al., 2006; Phillips and Dudík,

207 2008). The MaxEnt model features impose varying constraints on the relationship between the

208 occurrences and user input environmental variables and result in models of varying complexities. It is

209 important to remember that even if multiple feature classes are allowed for model-building, not all

210 classes will necessarily be incorporated in the final model. The default MaxEnt setting for feature class,

211 called "auto features," applies the class or classes estimated to be appropriate for the particular sample

212 size of occurrence records based on extensive tuning experiments (Phillips and Dudík, 2008). The use of

213 complex feature settings allows MaxEnt to produce a model that is more sensitive to details of a species'

214 environmental tolerances, however, complex feature classes can also lead to over-fit models. Phillips

215 and Dudík (2008) selected the following feature classes for continuous variables as default for the

216 corresponding occurrence record sample sizes: all feature classes for at least 80 occurrence records; L, Q

217 and $\mathrm{H}$ for sample sizes 15 to 79; $\mathrm{L}$ and $\mathrm{Q}$ for 10 to 14 records; only L for below 10 records (Phillips and

218 Dudík, 2008). These settings were subsequently implemented in the "auto features" of MaxEnt.

219 However, as recommended by Shcheglovitova and Anderson (2013) to reduce model overfitting,

220 SDMtoolbox does not limit feature class use by the number of occurrence records and allows users to

221 compare models created from 5 different combinations of feature classes: 1. L; 2. L \& Q; 3. H; 4. L, Q \&

$222 \mathrm{H} ;$ 5. L, Q, H, P \& T. For the fifth group of feature class combinations, in SDMtoolbox v2 the threshold

223 feature class $(T)$ can be enabled or disabled in accordance with the current MaxEnt 3.4.1

224 recommendations (where $\mathrm{T}$ is disabled) or previous versions (where $\mathrm{T}$ is enabled). The $\mathrm{C}$ class is reserved

225 for categorical variables and independently applied to associated layers in all 5 feature class

226 combinations. In addition to the methods implemented in SDMtoolbox, we urge users to view of

227 responses curves in their final model and evaluate if they are biologically logical.

228

Spatial jackknifing (or geographically structured k-fold cross-validation) tests and evaluates performance of spatially segregated localities. The last step of model parameterization SDMtoolbox implements spatial jackknifing. To do this SDMtoobox splits the landscape into 3-5 regions based on Voronoi polygons (a polygon whose interior consists of all area inside each is closest to an individual occurrence record) and spatial clustering of occurrence points (Fig 1G). Models are calibrated with all permutations of the groups using occurrence points and background data from n-1 spatial groups and then evaluated with the withheld group (Fig 1H). Spatial jackknifing has demonstrated clear advantages over random 
235

236

237

238

239

240

241

242

243

244

245

246

247

248

249

250

251

252

253

254

255

256

257

258

259

260

261

262

263

264

265

266

267

268

269

270

sampling of test/training occurrence data (as is common practice and the default setting in MaxEnt). In experiments on the effects of these two treatments, randomly partitioned occurrence datasets produced inflated estimates of performance and led to over-fit models (Radosavljevic \& Anderson 2014). Under the spatial jackknifing approach, increasing the regularization multiplier did not sufficiently counteract the effects of the strong spatial bias in the localities used for model calibration (artificially inserted into their experimental approach; Radosavljevic \& Anderson 2014). In contrast, the spatial jackknifing approach was shown to sidestep problems of the artificial spatial bias (and any corresponding environmental biases) and allow for detection of overfitting to environmental biases that differed among the spatial partitions (Phillips, 2008). Spatial jackknifing, evaluation of multiple feature classes and multiple regularization parameters are implemented within the Run MaxEnt: Spatial Jackknifing tool.

\section{CONCLUSIONS}

The scripts in SDMtoolbox 2.0 streamline many geospatial analyses and simplify GIS processes associated with analyzing biological datasets. SDMtoolbox 2.0 can dramatically reduce the repetitive and time-consuming analyses commonly associated with species distribution modeling: data pre-processing, modeling parametrization, model evaluation, and post-SDM analyses. This release of SDMtoolbox allows researchers to use the most current ESRI software and reduces the amount of time that would be spent developing common solutions. The latest version of SDMtoolbox, a user guide, and example data are freely available at http://www.sdmtoolbox.org. For questions or suggestions regarding SDMtoolbox 2.0, go to our google group

\section{https://groups.google.com/forum/\#!forum/sdmtoolbox}

or email sdmtoolbox.help@gmail.com.

\section{References}

Anderson, R.P. (2003) Real vs. artefactual absences in species distributions: tests for Oryzomys albigularis (Rodentia: Muridae) in Venezuela. Journal of Biogeography, 30, 591-605.

Anderson, R.P. \& Gonzalez, J.I. (2011) Species-specific tuning increases robustness to sampling bias in models of species distributions: an implementation with MaxEnt. Ecological Modelling, 222, 2796-2811.

Anderson, R. P. and Raza, A. 2010. The effect of the extent of the study region on GIS models of species geographic distributions and estimates of niche evolution: preliminary tests with montane rodents (genus Nephelomys) in Venezuela. -Journal of Biogeography 37:1378-1393. 
271

272

273

274

275

276

277

278

279

280

281

282

283

284

285

286

287

288

289

290

291

292

293

294

295

296

297

298

299

300

301

302

303

304

305

306

307

308

309

310

311

312

313

314

315

316

317

318

Barbet-Massin, M., Jiguet, F., Albert, C. H. and Thuiller, W. 2012. Selecting pseudo-absences for species distribution models: how, where and how many? -Methods in Ecology and Evolution 3:327-338.

Barve, N., Barve, V., Jiménez-Valverde, A., Lira-Noriega, A., Maher, S.P., Peterson, A.T., Soberón, J. and Villalobos, F. 2011. The crucial role of the accessible area in ecological niche modeling and species distribution modeling. -Ecological Modelling 222 (11): 1810-1819.

Brown J.L. 2014, SDMtoolbox: a python-based GIS toolkit for landscape genetic, biogeographic, and species distribution model analyses. -Methods in Ecology and Evolution 5(7):694-700.

ESRI 2017. ArcGIS Desktop and Spatial Analyst Extension: Release 10.5 Redlands, CA: Environmental Systems Research Institute.

Laffan, S.W., Lubarsky, E. and Rosauer, D.F. 2010. Biodiverse, a tool for the spatial analysis of biological and related diversity. -Ecography 33: 643-647 (version 1.0).

Lobo, J.M., Jiménez-Valverde, A. \& Real, R. (2008) AUC: a misleading measure of the performance of predictive distribution models. Global Ecology \& Biogeography, 17, 145-151.

McRae, B. H. and Beier, P. 2007. Circuit theory predicts gene flow in plant and animal populations. Proceedings of the National Academy of Sciences 104(50): 19885-19890.

Mislher B.D., Knerr N., Gonzalez-Orozco C.E., Thornhill A.H., Laffan S.W., and Miller J.T. 2014.

Phylogenetic measures of biodiversity and neo- and paleo-endemism in Australian Acacia. -Nature Communications 5: 4473.

Merow, C., Smith, M.J., and Silander, J.A. 2013. A practical guide to MaxEnt for modeling species' distributions: what it does, and why inputs and settings matter. -Ecography 36:1058-1069.

Peterson, A.T., Soberón, J., Pearson, R.G., Anderson, R.P., Martínez-Meyer, E., Nakamura, M. \& Araujó, M.B. (2011) Ecological Niches and Geographic Distributions. Monographs in Population Biology, 49. Princeton University Press, Princeton, New Jersey, USA.

Phillips, S.J., Anderson, R.P. \& Schapire, R.E. (2006) Maximum entropy modeling of species geographic distributions. Ecological Modelling, 190, 231-259.

Phillips, S.J. \& Dudík, M. (2008) Modeling of species distributions with MaxEnt: new extensions and a comprehensive evaluation. Ecography, 31, 161-175.

Phillips S.J., Dudík M., Schapire, R. 2017. [Internet] MaxEnt software for modeling species niches and distributions (Version 3.4.1). Available from url:

http://biodiversityinformatics.amnh.org/open_source/MaxEnt/. Accessed on 2017-5-3.

Radosavljevic, A. \& Anderson, R.P. (2014) Making better MaxEnt models of species distributions: complexity, overfitting and evaluation. Journal of Biogeography, 41, 629-643.

Ray, N. 2005. PATHMATRIX: a geographical information system tool to compute effective distances among samples. -Molecular Ecology Notes 5(1):177-180. 
319

320

321

322

323

324

325

326

327

328

329

330

331

332

333

334

335

336

337

338

339

340

341

342

343

344

345

346

347

348

349

350

351

Warren, D.L. \& Seifert, S.N. (2011) Ecological niche modeling in MaxEnt: the importance of model complexity and the performance of model selection criteria. Ecological Applications, 21, 335-342.

Figure 1. Visual Overview of Using SDMtoolbox to model in MaxEnt. SDMtoolbox box has tools to facilitate input and processing occurrence and environmental data: A. Convert CSV or XLS files to shapefiles. B. Clipping environmental layers to spatial extent C. \& D. Conversion from raster formats to ASCII for MaxEnt. SDMtoolbox also reduces spatial biases in occurrence record by spatially rarefying the points (a.k.a. spatial filtering) to reduce clusters of points. E. Areas of high spatially autocorrelated occurrence records that were removed during spatial rarefying. Blue to orange colored polygons depict low to high levels of spatial autocorrelation existing in occurrence records prior to spatial rarefying. F. The spatial filtering process can be done with a single spatial filter or up to five spatial filters to account for topographic and climatic heterogeneity. For example, in areas of high climate heterogeneity points could be filtered at a smaller area and in areas of low climate heterogeneity at larger spatial scales (i.e., $5 \mathrm{~km}^{2}$ for areas of high and $15 \mathrm{~km}^{2}$ for areas of low heterogeneity). G. One way SDMtoolbox minimizes model overfitting of each species distribution model it creates is by carefully controlling the background selection using bias files. SDMtoolbox provides several methods for being more selective in the choice of background points in MaxEnt: i) distance from observation points, ii) buffered local adaptive convex-hull of observations (a flexible way to create cluster of smaller convex polygons), and iii) buffered minimumconvex polygon of observation points. $\mathrm{H}$. Spatial jackknifing tests and evaluating performance of spatially segregated localities. SDMtoobox splits the landscape into 3-5 regions based on spatial clustering of occurrence points $(\mathrm{Hi})$ and classification of clusters into Voronoi polygons (Hii-iii). Users can have between 3-5 spatial random (Hiv) or segregated groups (Hv). Models are calibrated using permutations of training occurrence data from n-1 spatial groups, and then are evaluated with the withheld spatial group (I). Here $k=3$ and each group was label as $A, B, C$. Models were trained with points from areas two areas, then evaluated with points from the area not included in training. This process continues until models are evaluated with point from each spatial area (e.g., A, B or C) and trained with points from all other areas (e.g., $A B, A C$ or $B C$, respectively). 


\section{Table 1 (on next page)}

Table 1. Major differences between SDMtoolbox V1 and V2 
1

2 Table 1. Major differences between SDMtoolbox V1 and V2

\begin{tabular}{|l|l|l|}
\hline Feature & SDMtoolbox V1 & SDMtoolbox V2 \\
\hline Compatibility with ArcGIS 10.3-10.5 & & X \\
\hline Input Parameters Output As File & X \\
\hline $\begin{array}{l}\text { Improved user performance, Python code } \\
\text { is optimized, expanded user-guide }\end{array}$ & $\mathrm{X}$ \\
\hline $\begin{array}{l}\text { Complete compatibility with the new open } \\
\text { source version of Maxent (version 3.4) }\end{array}$ & & $\mathrm{X}$ \\
\hline Total Tools & 59 & 79 \\
\hline
\end{tabular}

3 
Table 2 (on next page)

Table 2. New Tools in SDMtoolbox v2.0 


\begin{tabular}{lll}
\hline Tool Subgroup and Name & Function & Numbers of \\
\hline
\end{tabular}

\section{Biodiversity Measurements}

- CANAPE categorization

- Quickly reclassify significance

from randomizations

-Runs categorizations of neo- and paleo-endemism on grids output from Biodiverse

-Uses data from Biodiverse to randomize and reclassify significance

\section{Landscape Connectivity}

- Create Pairwise Distance Matrix -Creates distance matrices showing both the least cost path (LCP) and the along path cost of the LCP

\section{SDM Tools}

- Split binary SDM by input clade relationship

-Sample by Buffered Local

Adaptive Convex-Hull
-Splits a binary SDM by input user clade relationships. Can only be done with 2-10 clade Groups

-Limits selection of background points to area inside a buffered regional convex-hull created through species occurrences

\section{Basic Table, Shapefile, and Raster Tools}

-Project Shapefiles to User

-Projects entire folder of shapefiles to any input projection

Specified Projection (folder)

- Define Projection (folder)

- Polygon to Raster (folder)

-Used to define the projection of any input (shapefile or raster)

-Converts polygon input into a raster format

-Converts all NetCDF (.nc) files to raster 
- Define NoData Value (folder)

-Advance Upscale Grids (folder) -Export JPEGs of all open files -Export Images of All Color Permutation of a $R G B$ raster -Sample raster values at input localities (folder)

-Increase Raster Extent/Snap All Raster to Same Extent (folder)
-Redefines NoData value in rasters. Used to fix an error when creating rasters where the NoData value is changed

-Upscales all grids in folder to a coarser resolution

-Exports JPEGS of all files in the map viewer

-Exports images of all color permutations of a RGB raster

-Samples the values of TIFF rasters at the locations input. Allows field names to be up to 50 characters

-This tool will increase or decrease spatial extent of all input rasters 


\section{Figure 1}

Figure 1. Visual Overview of Using SDMtoolbox to model in Maxent.

SDMtoolbox box has tools to facilitate input and processing occurrence and environmental data: A. Convert CSV or XLS files to shapefiles. B. Clipping environmental layers to spatial extent C. Conversion from raster formats to ASCII for Maxent. SDMtoolbox also reduces spatial biases in occurrence record by spatially rarefying the points (a.k.a. spatial filtering) to reduce clusters of points. E. Areas of high spatially autocorrelated occurrence records that were removed during spatial rarefying. F. The spatial filtering process can be done with a single spatial filter or up to five spatial filters to account for topographic and climatic heterogeneity. For example, in areas of high climate heterogeneity points could be filtered at a smaller area, whereas areas of low climate heterogeneity at larger spatial scales (i.e., $5 \mathrm{~km}^{2}$ and $15 \mathrm{~km}^{2}$ respectively). G. One way SDMtoolbox minimizes model overfitting of each species distribution model it creates is by carefully controlling the background selection using bias files. SDMtoolbox provides several methods for being more selective in the choice of background points in Maxent: i) distance from observation points, ii) buffered local adaptive convex-hull of observations (a flexible way to create cluster of smaller convex polygons), and iii) buffered minimum-convex polygon of observation points. H. Spatial jackknifing tests and evaluating performance of spatially segregated localities. SDMtoobox splits the landscape into 3-5 regions based on spatial clustering of occurrence points (Hi) and classification of clusters into Voronoi polygons (Hii-iii). Users can have between 3-5 spatial random (Hiv) or segregated groups (Hv). Models are calibrated using permutations of training occurrence data from n-1 spatial groups, and then are evaluated with the withheld spatial group (I). Here $k=3$ and each group was label as $A, B, C$. Models were trained with points from areas two areas, then evaluated with points from the area not included in training. This process continues until models are evaluated with point from each spatial area (e.g. A, B or C) and trained with points from all other areas (e.g. BC, AC or BC, respectively). Peer] reviewing PDF | (2017:06:18701:2:0:NEW 13 Oct 2017) 


\section{Ban:}

OCCURRENCE \& ENVIRONMENTAL DATA PROCESSING

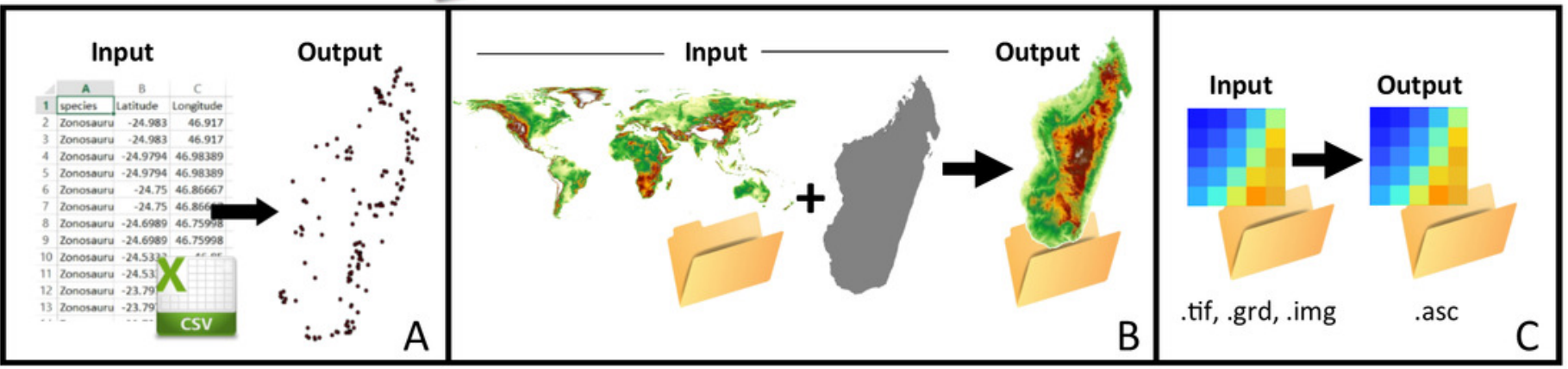

RAREFYING OCCURRENCE RECORDS



BACKGROUND SELECTION VIA BIAS FILES

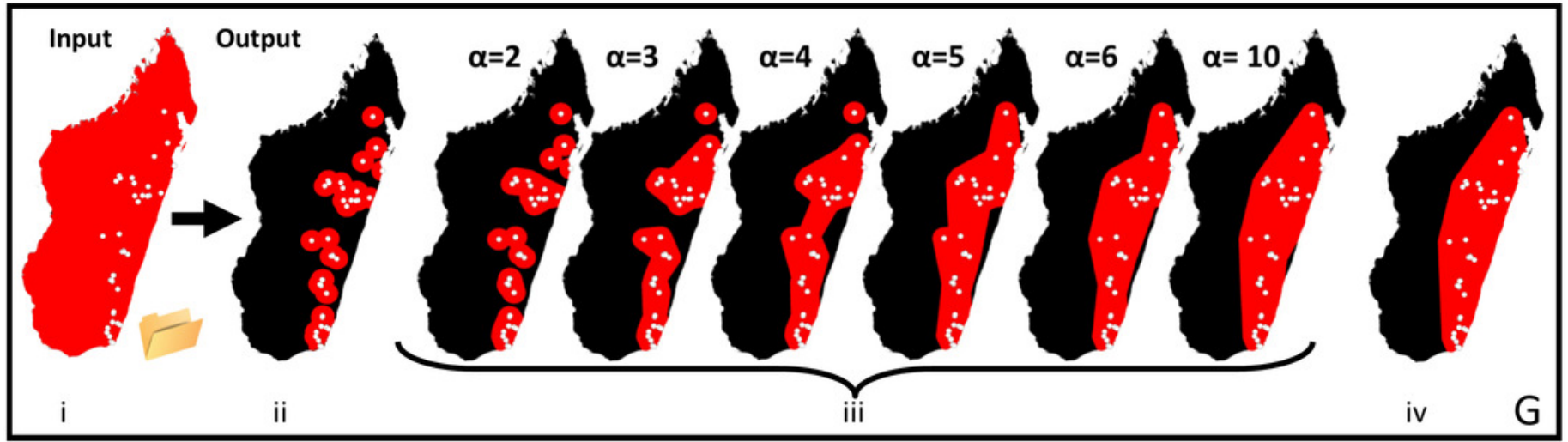

SPATIAL JACKKNIFING

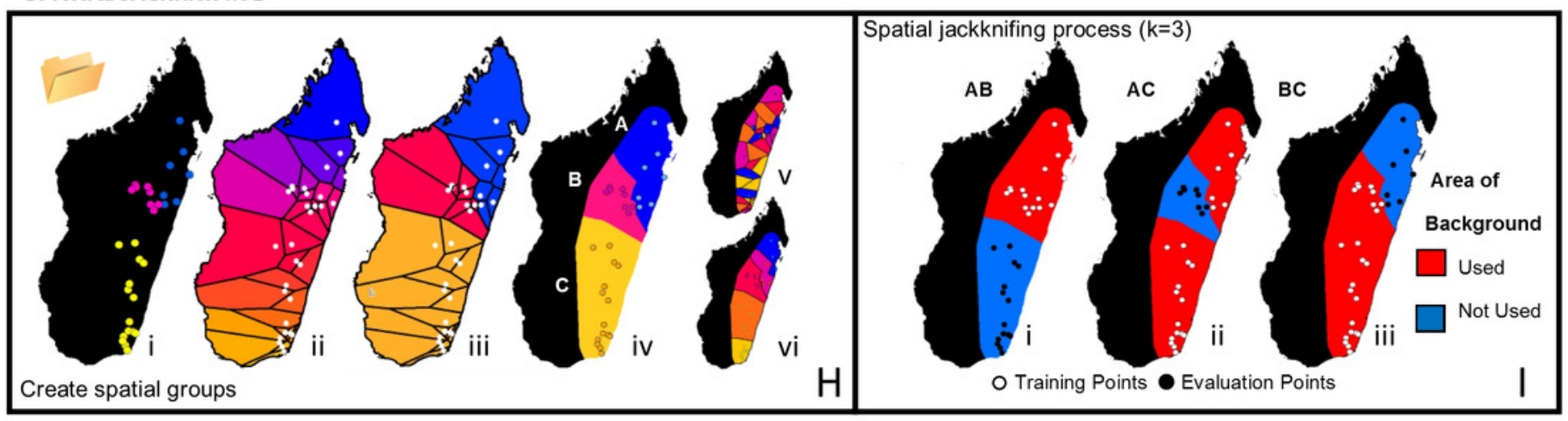

